\title{
Que "diferença faz a diferença" na recuperação da anorexia nervosa?
}

\section{Which "difference does make a difference" in recovery of anorexia nervosa?}

\author{
Maria Xavier de Araújo ${ }^{1}$, Margarida Isabel Rangel Santos Henriques ${ }^{2}$ \\ 1 Licenciada em Psicologia, terapeuta familiar, bolsista de investigação pela Fundação para a Ciência e Tecnologia. \\ 2 Doutora em Psicologia, terapeuta familiar, professora auxiliar da Faculdade de Psicologia e Ciências da Educação da Universidade do Porto, Portugal.
}

Recebido: 30/10/2009 - Aceito: 26/7/2010

\section{Resumo}

Contexto: As dificuldades inerentes ao tratamento da anorexia nervosa são bem conhecidas e é, ainda hoje, predominante a concepção da anorexia nervosa enquanto doença crônica. Contudo, diversos estudos mostram não só que a recuperação é possível como também que há inclusivamente mulheres que se recuperam espontaneamente, sem terem sido sujeitas a tratamento. Objetivo: Este estudo pretende, assim, rever a literatura existente relativamente a fatores que contribuíram para a recuperação na anorexia nervosa, quer relacionados com o tratamento quer com extratramento. Método e resultados: Para tal, a partir da revisão de 13 estudos existentes sobre a perspectiva de ex-pacientes acerca do que contribuiu para a recuperação, este artigo irá pôr em destaque que "diferenças fizeram a diferença", bem como em que medida os estudos existentes permitem uma compreensão de como essas diferenças podem fazer a diferença. Conclusão: Conclui-se que, apesar de a investigação estar, sobretudo, centrada na compreensão dos fatores de tratamento mais úteis, muitas ex-pacientes parecem destacar mais a utilidade dos fatores extratratamento, nomeadamente a importância das relações na manutenção e resolução do problema. Os mesmos fatores são considerados prejudiciais e/ou úteis para diferentes entrevistadas, o que remete para a complexidade do fenômeno da recuperação que ainda carece de mais investigação.

Araújo MX, Henriques MIRS / Rev Psiq Clín. 2011;38(2):71-6

Palavras-chave: Anorexia nervosa, recuperação, fatores de mudança.

\begin{abstract}
Background: The difficulties in the treatment of anorexia nervosa are well known and there is still today a dominant conception of anorexia nervosa as a chronic illness. Nevertheless, several studies show not only that recovery is possible but also that there are women who recover spontaneously, even without medical treatment. Objective: This study intends to review the existing literature on the factors that contributed to the recovery in anorexia nervosa, whether or not related to treatment. Methods and results: From the review of 13 existing studies, on the perspective of former patients about what contributed to the recovery, this article highlights which "differences did make a difference" and analyzes how these differences can actually make the difference. Conclusion: The authors conclude that although research had been mainly focused on understanding the most important medical treatment factors, many former patients emphasize the usefulness of extra-treatment factors, namely the importance of relationships in the maintenance and resolution of the problem. The same factors are considered harmful and/or useful by different respondents, which bring us to the complexity of the recovery phenomenon that still requires further research.
\end{abstract}

Araújo MX, Henriques MIRS / Rev Psiq Clín. 2011;38(2):71-6

Keywords: Anorexia nervosa, recovery, turning-points.

\section{Introdução}

O fenômeno da anorexia nervosa (NA) é amplamente investigado, não só dado o acréscimo da sua incidência na atualidade, mas também pela significativa morbidade e mortalidade a ela associadas. Apesar da crescente investigação, nos últimos 50 anos, poucos progressos foram atingidos no desenvolvimento de novos e eficazes tratamentos ${ }^{1}$, sendo ainda atualmente considerada por alguns autores como uma das mais frustrantes formas de psicopatologia ${ }^{2}$. A investigação demonstra que o resultado do tratamento nem sempre é positivo, as taxas de drop-out do tratamento são muito altas ${ }^{3} \mathrm{e}, \mathrm{em}$ comparação com outras doenças mentais, a anorexia nervosa tem as mais altas taxas de mortalidade, variando entre $5 \%$ e $22 \% 4$.

Apesar da "habilidade e pertinaz resistência das doentes às propostas terapêuticas" ${ }^{5}$, a recuperação na anorexia nervosa é possível, ainda que se afigure como um processo difícil e moroso, muitas vezes implicando de cinco a oito anos de progresso lento, de avanços e recuos, de recaídas, até um movimento último no sentido da saúde física e mental ${ }^{6,7}$. Efetivamente a recuperação tem sido uma evidência de vários estudos, ainda que os resultados demonstrem grande variabilidade. Herzog et al. ${ }^{8}$, por exemplo, encontraram taxas de que variam entre $17 \%$ e $77 \%$ nos quatro anos ou mais após tratamento, enquanto Steinhausen ${ }^{9}$ encontrou variações de 0 a $92 \%$. A dificuldade de chegar a taxas consensuais de recuperação deve-se a inúmeros fatores. Couturier e Lock ${ }^{10}$ concluíram que as taxas de recuperação variam amplamente dependendo dos critérios específicos usados. Os estudos baseiam-se em diferentes definições de recuperação e, além disso, em tempos de follow-up diferentes, bem como em amostras com características diferentes e tipos de tratamento diferentes ${ }^{10}$.

Para além das dificuldades de reunir consenso relativamente às taxas de recuperação, ainda persistem entre os profissionais de saúde e até entre os próprios pacientes e familiares concepções da anorexia nervosa enquanto doença crônica ${ }^{11}$. No entanto, o prognóstico aponta para uma porcentagem significativa de pacientes que se recupera totalmente (47\%), outra que atinge recuperação parcial $(33,5 \%)$, enquanto apenas $20 \%$ permanecem crônicas 9 . Mas, segundo Pinzon e Nogueira ${ }^{12}$, esses números não podem ser encarados como muito animadores, pois, mesmo mantendo peso e menstruações normais, grande parte das pacientes com AN mantém alterações físicas, psicológicas ou sociais.

Ê também conhecida a lacuna existente na investigação sobre os melhores tipos de tratamento, as combinações mais adequadas de componentes de tratamento em diferentes fases da doença ou sobre a duração necessária do tratamento com vista à recuperação das mulheres com anorexia nervosa ${ }^{13,14}$. Kaplan ${ }^{1}$, em uma revisão exaustiva dos estudos sobre os tratamentos psicológicos empiricamente validados 
na anorexia nervosa nas últimas três décadas, identificou menos de 20 estudos que avaliam o impacto de vários tipos de psicoterapia no tratamento da anorexia nervosa e concluiu que a evidência da eficácia das intervenções, com exceção da terapia familiar para os pacientes mais jovens, permanece questionável.

Há também muito pouca investigação que olhe para a recuperação, do ponto de vista do paciente, e para os fatores que levam à recuperação a longo prazo da anorexia nervosa ${ }^{15}$. Essa lacuna na investigação, quer relativamente ao estudo dos tratamentos e componentes de tratamento mais eficazes, quer relativamente ao ponto de vista do paciente no que diz respeito à recuperação, torna difícil a resposta à questão: O que contribui para a recuperação? Ou como diria Bateson: Que diferença faz a diferença na recuperação da anorexia nervosa? Sabe-se de antemão que para a recuperação não só contribuem fatores relacionados com o tratamento, como também aspectos da vida e das relações, já que existe uma percentagem de mulheres que se recuperam espontaneamente sem serem sujeitas a tratamento ${ }^{16}$.

Dada a evidência de recuperação de anorexia nervosa, ainda que as taxas e as concepções não sejam consensuais, e tendo em consideração as dificuldades de tratamento existentes, urge pôr em destaque os casos bem-sucedidos, bem como compreender o que contribuiu para a recuperação do ponto de vista das ex-pacientes. Aliás, tendo em conta a importância cultural do mito e o significado da autotransformação, Garrett ${ }^{17}$ propõe que quantas mais histórias e modelos de recuperação estiverem disponíveis, mais provável a recuperação se torna.

Assim, este artigo pretende precisamente propor uma mudança do objeto de estudo, por meio de um olhar mais centrado nas soluções, procurando compreender a recuperação, em vez de um olhar etiológico, que explica e se centra nas causas da doença. Tal como afirma Garrett ${ }^{17}$, explorar a solução para um problema pode ser mais valioso que investigar a sua etiologia, já que a solução pode clarificar a causa e, se não o fizer, pode sugerir que a causa não é muito relevante. Propõe-se, desse modo, uma análise integradora da investigação existente sobre fatores úteis à recuperação, do ponto de vista das mulheres recuperadas.

\section{Método}

Realizou-se uma pesquisa utilizando a base de dados EBSCOhost, com os termos de busca: "anorexia nervosa" e "recovery" ou "change". Em virtude do reduzido número de artigos encontrados que se debruçassem sobre fatores de recuperação ou sobre o processo de mudança na anorexia nervosa $(\mathrm{N}=6)$, procurou-se também, na base de dados ProQuest, teses de investigação sobre o tema $(\mathrm{N}=5)$. E, adicionalmente, foram selecionados outros artigos por pesquisa ativa, a partir das referências bibliográficas presentes nas pesquisas encontradas.

\section{Resultados e discussão dos resultados}

A partir da pesquisa realizada, foram identificados no total 13 estudos, publicados entre 1985 e 2009, todos eles focados na percepção das próprias mulheres com diagnóstico passado de anorexia nervosa e, em todos os estudos, incidindo-se particularmente sobre os fatores que foram úteis para a recuperação. Esses estudos incidiram sobre uma amostra que variou entre os 7 e os 62 participantes, com uma média de 24 participantes entrevistadas com diagnóstico anterior de anorexia nervosa. $\mathrm{O}$ tempo de recuperação selecionado pelos diferentes estudos foi bastante variável (recuperadas desde há seis meses até há pelo menos dez anos), e todas as amostras apenas contemplaram participantes com mais de 18 anos.

Da análise desses 13 estudos, procedeu-se a uma sistematização do tipo de fatores referidos como tendo sido úteis à recuperação, principais fatores úteis à recuperação, organizando-os segundo as categorias e subcategorias a seguir apresentadas:
A. Modalidades terapêuticas
- Intervenção em grupo
- Intervenção familiar
- Psicoterapia individual

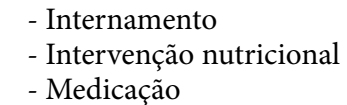

B. Fatores terapêuticos

- Motivação para mudar

- Relação terapêutica

- Segurança e estrutura

- Superar um trauma passado

- Trabalho com relação às emoções

C. Fatores extratratamento

- Relações significativas

- Separação da família

- Conhecer outras pacientes

- Conhecer jovens recuperadas

- Ativismo social

- Compromisso espiritual

- Sucesso acadêmico/profissional

- Fatores individuais (motivação, determinação, autoaceitação)

\section{A. Modalidades terapêuticas}

Atualmente, os tratamentos para a anorexia nervosa incluem muitas vezes mais do que um componente ou modalidade de intervenção. Simmons ${ }^{15}$, Tozzi et al. ${ }^{18}$, Hsu et al. ${ }^{19}$, Beresin et al. ${ }^{11}$ e Maine ${ }^{20}$ procuraram perceber, considerando o ponto de vista de mulheres recuperadas, quais os componentes do tratamento mais úteis à recuperação.

Assim, a intervenção em grupo foi considerada pelas entrevistadas um componente positivo na medida em que permitia às participantes experimentarem um sentimento que apoio, retirando-as, assim, do isolamento em que muitas vezes elas acabam por cair. A intervenção em grupo permitiu, segundo as mulheres recuperadas, o contato com outras pessoas que as entendem, dando-lhes a sensação de "estarem todas no mesmo barco"15. Por outro lado, as entrevistas realizadas por Beresin et al. ${ }^{11}$ com 13 mulheres, revelam que, apesar de as participantes destacarem os benefícios da intervenção em grupo quanto à redução da vergonha, do medo e da culpa, elas contrabalançam essa avaliação com a desvantagem de, por vezes, os grupos favorecerem a competição, bem como a aprendizagem de novos comportamentos para emagrecer. Simmons ${ }^{15}$ afirma que nem todos os grupos são considerados pelas entrevistadas como úteis, nomeadamente os grupos para lidar com a imagem corporal ou sexualidade, que são percebidos como muito difíceis para as participantes e pouco úteis, embora algumas participantes sintam que tocam em aspectos fundamentais relacionados com a doença. No mesmo sentido, quatro de seis entrevistadas por Maine ${ }^{20}$ que tiveram terapia em grupo sentiram-se desconfortáveis e expostas, tendo achado pouco útil essa modalidade de intervenção. Todas as entrevistadas referiram-se à intervenção em grupo com pacientes com a mesma patologia. Contudo, permanece a dúvida se a intervenção em grupo poderia ser útil caso o grupo englobasse diferentes patologias, tornando a competição e a aprendizagem de novos comportamentos para emagrecer menos provável, ou mesmo se o grupo incluísse ex-pacientes, de forma a tornar possível a identificação com percursos com um final diferente, o que poderia alargar a consciência de algumas pacientes relativamente ao leque de possibilidades e alternativas de vida que cada uma pode escolher.

A terapia familiar afigura-se como uma modalidade de intervenção muito usada nesse tipo de patologia e é vista como um componente útil do tratamento pelas ex-pacientes ${ }^{11,19,20}$, na medida em que permitiu às mulheres entrevistadas melhor compreensão dos pais, bem como resultados positivos na melhoria da relação entre os membros ${ }^{11}$. No estudo de Maine ${ }^{20}$, duas entrevistadas que foram acompanhadas em terapia familiar destacam a melhoria da comunicação, a descoberta de segredos familiares, bem como a possibilidade que devolveu à paciente de ser capaz de ter as suas próprias opiniões como efeitos positivos da terapia familiar. Para além disso, algumas entrevistadas que não tiveram terapia familiar acharam que poderia ter sido útil. Um interessante testemunho 
presente no estudo de Maine ${ }^{20}$ aponta para o fato de a anorexia nervosa ser um fenômeno inteiramente familiar, tornando explícita a necessidade de terapia familiar. Contudo, a terapia familiar parece não ser considerada útil quando ocorre abuso sexual na família, já que a família permanece nesses casos em silêncio ${ }^{15}$. Outro aspecto negativo destacado pelas entrevistadas foi o fato de esse tipo de intervenção ter levado a que os pacientes expressassem raiva ${ }^{11}$. Se esse aspecto é apontado como negativo pelas próprias pacientes, para um terapeuta esse aspecto pode ser interpretado como uma mudança positiva no modus operandis da família, permitindo ao paciente a sua expressão emocional em detrimento do evitamento de conflitos, muitas vezes frequente nessas famílias. Seria interessante o confronto dessas duas perspectivas, desses dois olhares, a fim de esclarecer até que ponto esse tipo de expressão emocional em família pode ser útil ou prejudicial, tendo em conta a forma como essa revelação pode ser trabalhada nas sessões subsequentes. Assim, permanece a dúvida se a expressão emocional na família é prejudicial por si, ou é apenas negativa quando a terapia não permite a ressignificação desses sentimentos, de forma que eles se tornem passíveis de existir sem porem em risco a estrutura familiar.

A psicoterapia individual foi outro dos fatores destacados como importante para a recuperação ${ }^{18-20}$. O estudo de Beresin et al. ${ }^{11}$, a partir das entrevistas que realizaram com 13 mulheres, concluiu que a psicoterapia individual foi relatada como a componente do tratamento mais útil e simultaneamente mais prejudicial. A utilidade estaria relacionada com a importância do confronto e dos limites e a importância de determinadas qualidades do terapeuta, nomeadamente, ser honesto, consistente, flexível, empático, não crítico. Sendo os testemunhos uma boa experiência de terapia, eles permitiram às pacientes aumentarem o conhecimento acerca de si próprias, aumentarem a sua autoconfiança e fortalecerem a sua capacidade de confiar nos seus próprios sentimentos e ideias. Por outro lado, Simmons ${ }^{15}$ destaca um aspecto comum a todas as suas entrevistadas: o fato de acharem que a psicoterapia individual falhou na medida em que não as "preparou o suficiente para as situações da vida real", ou seja, já que o tratamento decorre num ambiente protegido, isso faz com que não as ajude a lidar com estímulos que muitas vezes são responsáveis pelas recaídas. Este, aliás, é um argumento frequentemente utilizado como desvantagem do tratamento em internamento, que permite modelar comportamentos num ambiente protegido que nem sempre são transferidos para a vida real. No entanto, na prática clínica, assistimos muitas vezes a jovens que após o internamento decidem adotar para o seu dia a dia precisamente as mesmas rotinas e horários do período em que estiveram internadas, o que mostra que não são apenas os contextos que determinam os comportamentos, mas também o próprio protagonismo assumido pelas pacientes individualmente.

Efetivamente, uma parte das mulheres desses estudos recebeu também tratamento em regime de internamento. No estudo de Beresin et al. ${ }^{11}$, foi dado destaque à hospitalização, vista como um alívio e um período de segurança. Também as mulheres entrevistadas por Hsu et al. ${ }^{19}$ destacaram esse como um dos fatores importantes para a recuperação, podendo ser inclusive um ponto de viragem importante para a recuperação. Maine ${ }^{20}$ chegou à mesma constatação para algumas das entrevistadas, contudo outras consideraram o internamento como prejudicial na medida em que se sentiram tratadas como crianças, em que tudo o que "fizeram foi alimentá-las" ${ }^{20}$. Esses dois argumentos parecem ilustrar um dilema, muito frequente na anorexia nervosa, que radica no conflito entre o desejo de proteção e segurança, por um lado, e o desejo de autonomia, por outro. Se o internamento lhes proporciona uma estrutura segura, satisfazendo-lhes as necessidades de proteção e segurança, é também um contexto que lhes limita a liberdade e o próprio protagonismo, algo que muitas vezes essas mulheres tanto desejam e, simultaneamente, receiam.

Pettersen e Rosenvinge ${ }^{21}$, a partir de entrevistas com 48 mulheres sobre fatores úteis para o processo de recuperação, concluíram ainda a importância, entre muitos outros aspectos, do seguimento de um plano de nutrição definido pelo clínico, no sentido de prevenção de recaídas. Algumas das entrevistadas por Simmons ${ }^{15}$ referiram igualmente que os programas que não tivessem um componente forte nutricional comportariam um grande déficit. No entanto, se há evidências de que o tratamento nutricional promove a regularização alimentar, há também evidências de que alguns comportamentos alimentares, como a sensação de incompetência ao lidar com os alimentos, permanecem ${ }^{22}$. A medicação também é destacada neste estudo como útil para regular a fome e ultrapassar crises emocionais. Contudo, no estudo de Beresin et al. ${ }^{11}$, esta é considerada como pouco útil.

Em jeito de síntese, as diferentes modalidades terapêuticas parecem todas terem vantagens e desvantagens, não sendo claro que umas sejam mais úteis para a recuperação que as outras. Fica também evidente o interesse de explorar em que fase da recuperação cada modalidade foi ou poderia ter sido mais útil.

\section{B. Fatores terapêuticos}

Para além dos componentes do tratamento, alguns estudos procuraram debruçar-se sobre fatores terapêuticos, independentemente da modalidade de intervenção adotada, que pudessem ter sido úteis para a recuperação. Assim, um dos fatores destacados como muito importante para a recuperação foi a motivação para mudar. Essa motivação é vista como fundamental para ter uma experiência positiva do tratamento ${ }^{21}$. Um fator que contribui para essa motivação é, muitas vezes, sentirem que chegaram a um limite e já estão doentes e cansadas de estar doentes ${ }^{19,23,24}$, pela constatação do impacto negativo da doença nas suas vidas ${ }^{23}$, ou de um simples "acordar" 18. A ameaça de internamento ou de fim de tratamento, com consequente separação do terapeuta, também foi relatada como poderosa para o aumento de motivação para o tratamento 20 .

A relação terapêutica foi outro fator considerado como muito importante para a eficácia do tratamento ${ }^{21}$. No estudo de Simmons ${ }^{15}$, a relação com o terapeuta foi considerado o fator mais importante para a recuperação, sendo necessário confiar no terapeuta, ter a sua aceitação incondicional e empatia, a sua atitude de não julgamento, até porque em alguns testemunhos, durante muito tempo, o terapeuta foi considerado como o único amigo da paciente ${ }^{20}$.

Perceber preocupação, amor incondicional e aceitação não só por parte dos terapeutas, mas também por parte dos familiares e amigos, foi um dos aspectos considerados como fundamentais para a recuperação em 14 entrevistas realizadas com mulheres recuperadas ${ }^{23}$. Essas entrevistadas estabelecem uma conexão entre o peso e o sentimento de ser aceito, aprovado, amado. Apresentam, assim, o medo de ser julgado como algo que só é superado com a aceitação e preocupação dos outros, algo indispensável para a recuperação. Pelo contrário, a percepção de falta de apoio é relacionada negativamente com a recuperação.

Outros aspectos relacionados com o tratamento, independentemente do componente usado, e igualmente considerados importantes para a recuperação, foram a segurança e a estrutura. As mulheres entrevistadas por Simmons ${ }^{15}$ destacaram a importância da segurança relacionada com o ambiente físico de tratamento e com a forma como a participante se sentiu com o profissional. Se a falta de segurança faz com que os participantes permaneçam na doença porque tal é mais seguro, pelo contrário, a segurança permite-lhes tentar outras alternativas ${ }^{15}$. A estrutura do tratamento baseada em regras bem definidas também constituiu uma importante ajuda à recuperação na medida em que deu às ex-pacientes uma sensação de controle, importante para investirem na recuperação. Também o estudo de Federici e Kaplan ${ }^{24}$ destaca o sentimento de segurança no tratamento como um componente importante para a recuperação.

Outro aspecto destacado por este último estudo foi a importância de lidar com o trauma, já que $70 \%$ das participantes da amostra entrevistada tinham uma história de abuso sexual. As participantes sentiram que um trauma as fez sentir que a vida estava fora do controle delas e a doença lhes dava um falso sentido de controle. $\mathrm{Na}$ maioria dos casos, o trauma não foi abordado no tratamento, o que levou ao adiamento da recuperação. 
Tanto o estudo de Simmons ${ }^{15}$ como o de Esherick ${ }^{23}$ enfatizam a importância do trabalho com relação às emoções. Esherick ${ }^{23}$ destaca a importância de lidar com as emoções no sentido de passar de uma fase de desejo de mudança para uma fase de mudança efetiva. Relativamente aos fatores que encontrou que contribuem para um aumento do desejo de mudança, ele destacou: a) aperceber-se do amor incondicional, aceitação e preocupação da parte dos outros; b) tornar-se cada vez mais consciente e frustrado com o impacto negativo da anorexia nas suas vidas e c) ter esperança por um futuro melhor. No sentido da passagem de uma fase de desejo de mudança para a ação, ele considerou que é necessário o trabalho de determinados temas relacionados com os sentimentos e identidade pessoal. Assim, descreve três etapas fundamentais:

1. Fase da autodescoberta, que implica que os participantes tomem contato com os seus sentimentos e desenvolvam um sentido de si.

2. Fase da autoaceitação, em que os participantes aceitam os seus sentimentos e aceitam-se a si próprios.

3. Fase da autoexpressão, que possibilita aos participantes começarem a exprimir os seus próprios sentimentos aos outros e afirmarem-se no mundo de forma que se sintam verdadeiros com o novo sentido de si que desenvolveram.

\section{Fatores extratratamento}

Segundo Beresin et $a l .{ }^{11}$, as experiências fora da terapia são tão importantes quanto as experiências de terapia no processo de recuperação. Aliás, no estudo de Maine ${ }^{20}$, das 25 participantes apenas 5 encararam o tratamento como essencial para a recuperação. Assim, alguns estudos também identificaram fatores úteis à recuperação não relacionados com o tratamento.

Um dos fatores mais destacados como importante para a recuperação situa-se no contexto das relações significativas, nomeadamente as relações com a família. Assim, ir para a faculdade ou sair de casa foi considerado pelas entrevistadas como algo útil, já que lhes permitiu estar longe de um ambiente percebido como pouco saudável e permitiu-lhes conhecer novas pessoas, ter novas experiências que lhes deram uma nova esperança de que a vida delas poderia ser melhor ${ }^{23}$. Nessa fase das suas vidas, começaram a ter a sensação de que precisariam desistir um pouco da anorexia nervosa para poderem ter outras coisas que descobriram que queriam. Das 25 entrevistadas por Maine ${ }^{20}, 8$ referiram a separação dos familiares como crucial para a recuperação, quer por meio da hospitalização, quer pela entrada na faculdade, ou de um trabalho de verão ou uma decisão de ir para casa de outros familiares. Se para algumas entrevistadas o envolvimento da família não foi útil, daí a necessidade de separação da família que as ajudou a ganhar melhor entendimento da dinâmica familiar ${ }^{11}$, para outras o apoio da família e de amigos foi muito importante para a recuperação, na medida em que favoreceu a aceitação, o afeto e o sentimento de valer a pena, aumentando, assim, o seu autoconceito. A constatação da dor na família foi também um fator importante de recuperação para 15 de 25 entrevistadas ${ }^{20}$, tendo essa constatação precedido a decisão de ficarem melhor.

A recuperação nesses estudos foi, muitas vezes, conectada com o permitir-se a si própria estar próxima dos outros 24 . Uma relação de apoio por parte de um companheiro ${ }^{18}$ ou por parte dos amigos ${ }^{25}$ foi um dos fatores que contribuiu mais para a recuperação.

Outros fatores extratratamento referidos pelos estudos sistematizados foram conhecer outros pacientes ${ }^{18,21}$ e conhecer jovens recuperadas $^{21}$. Nesse sentido, Simmons ${ }^{15}$ refere a partilha de refeições com outras pacientes como algo positivo que permite a partilha de medos associados à alimentação. No estudo de Maine ${ }^{20}, 15$ de 25 mulheres entrevistadas sugeriram que falar com alguém recuperado poderia tê-las ajudado, e Esherick ${ }^{23}$, inclusive, relata o caso de uma entrevistada que, quando conheceu uma mulher recuperada, começou a ter esperança de que algum dia fosse se recuperar, ou seja, ao ouvir o testemunho da recuperada, apercebeu-se de que haveria uma terceira opção para a sua vida, para além das duas alternativas que até então via para si: aguentar em toda a sua vida a voz "atormentadora" da anorexia ou morrer.

Matusek ${ }^{26}$, a partir da análise de entrevistas com oito participantes, concluiu que o componente principal das suas histórias de recuperação emerge das formas de ativismo social e de um profundo compromisso espiritual. A recuperação a longo prazo necessita de mais do que relações sólidas, bom aconselhamento nutricional e psicoterapia. Na sua amostra, este caminho de ativismo social ou de compromisso social foi feito pelas participantes por meio do ensino religioso, ativismo em centros para mulheres, feminismo, trabalho social, práticas de meditação, leituras e escrita de livros. Para as entrevistadas, a recuperação pressupôs o envolvimento em alguma causa ou comunidade "maior que elas próprias". Também Simmons ${ }^{15}$ verificou que os participantes que tiverem um componente espiritual forte relataram também um plano de prevenção de recaídas forte, o que indicia que a fé pode estar a serviço da esperança e da força na recuperação. Garrett 17 também enfatizou a importância da espiritualidade para a recuperação, encarando, aliás, a anorexia nervosa como uma fase de separação necessária, num ritual de passagem, e a recuperação como a fase de reconexão, de voltar a estar em paz consigo próprio. Maine ${ }^{20}$ alerta, no entanto, para o fato de algumas participantes verem a religião como uma extensão da doença, na medida em que cria um sistema de regras semelhante à anorexia e exemplifica com um caso de uma mulher que só após o corte com esse sistema começou a recuperar-se.

Outro dos fatores destacados dos estudos é o sucesso acadêmico ou profissional, que pode contribuir para a recuperação, na medida em que suscite satisfação ${ }^{11,18}$.

Para além de fatores relacionais ou externos ao próprio, há ainda aspectos individuais considerados úteis para a recuperação. Federici e Kaplan ${ }^{24}$, Federici27 e Maine ${ }^{20}$ salientam a autoaceitação e o sentimento de valer a pena como fatores importantes. Esse fator parece estar muito relacionado com outro: o de tomarem consciência das suas emoções e aprenderem a ser tolerantes com elas ${ }^{24}$. Outros fatores individuais considerados úteis foram o aumento da autoestima, a determinaçãao ${ }^{18}$, a automotivação e o assumir da responsabilidade por si, traduzidos no reconhecimento das dificuldades e necessidade de se responsabilizarem por si próprias, pela mudança, o que contrasta com o que usualmente antes faziam, que era basearem as decisões nos desejos e comportamentos dos outros significativos ${ }^{20}$.

Como foi descrito, não só os fatores relacionados com o tratamento são referidos pelas mulheres recuperadas como tendo sido úteis à recuperação. Apesar de não sabermos em quantos casos de perturbações do comportamento alimentar os pacientes podem melhorar ou até se recuperar sem tratamento especializado ${ }^{28}$, dada a dificuldade em aceder a esse tipo de participantes, Woods ${ }^{16}$ aplicou um questionário, via e-mail, a 18 inquiridos, que relataram recuperação sem tratamento. Os participantes destacaram como fatores úteis para a recuperação fatores relacionais, nomeadamente o apoio da mãe; uma reação mais emotiva do pai, após um período de raiva e tensão; a ajuda do namorado e de um amigo, bem como o desejo interior de uma vida melhor após a constatação dos problemas de saúde decorrentes da doença.

\section{Conclusão}

"More than two decades later I realized that recovery was in many ways more complicated that the eating disorder itself." 29

Esta revisão evidencia a pouca investigação existente relativa à recuperação a partir de mulheres recuperadas, apesar da existência de uma vasta gama de investigações no âmbito da anorexia nervosa, nomeadamente de estudos que incidem sobre a sua etiologia. Contudo, atualmente, com as correntes pós-modernas, a ênfase na descoberta das leis das causas tem perdido algum terreno a favor da definição de soluções, considerando-se que há muitos fenômenos que iniciam por aleatoriedade e que depois são alimentados por conjugações de fatores, não tendo assim a solução que passar pela compreensão da sua etiologia. 
Foi também possível compreender que a recuperação se afigura como um processo complexo, para o qual contribuem não só fatores relacionados com o tratamento, mas também outros fatores não relacionados com o tratamento. Aliás, apesar do interesse dos investigadores em perceber quais os componentes de tratamento mais úteis, muitas ex-pacientes parecem valorizar mais a utilidade dos aspectos não relacionados com o tratamento para a recuperação. Esse resultado pode ser lido como uma tentativa por parte das ex-pacientes de recusarem a construção médica do fenômeno da recuperação, algo que também pode estar já em si relacionado com uma concepção não estritamente médica do problema. Aliás, não é raro a qualquer clínico confrontar-se com mulheres, e mesmo familiares, que negam inicialmente que o problema em causa se trata de uma doença mental. Com efeito, a concepção da recuperação pode estar diretamente relacionada com a concepção do problema, e para esse fenômeno, que se convencionou denominar por "anorexia nervosa", podem coexistir construções médicas com outras construções mais individuais.

Incidindo agora mais particularmente nos fatores de recuperação, um dos fatores não relacionados com o tratamento destacados pelos estudos foi a importância das relações, nomeadamente as familiares, para a manutenção ou para a resolução do problema. Curiosamente, os testemunhos parecem indicar que tanto um sentimento de apoio por parte de um familiar ou pessoa próxima como o distanciamento familiar parecem poder ser fatores úteis para a recuperação para diferentes entrevistadas. Ou seja, a família é simultaneamente passível de promover a manutenção do sintoma ou de contribuir para a sua resolução. Esse dado necessitaria de uma abordagem mais compreensiva para se perceber de que forma a família pode ter uma ou outra função, o que poderia passar por estudar a recuperação não apenas do ponto de vista das pacientes recuperadas, mas também a partir do ponto de vista familiar acerca da recuperação.

Os resultados ainda mostram que os mesmos fatores, como esse das relações familiares, ou outros como o da psicoterapia individual ou da intervenção em grupo, são encarados como prejudiciais e/ou como úteis para diferentes entrevistadas. Esse é um resultado interessante na medida em que nos faz crer que não é o fator em si que define a sua utilidade, mas antes o significado e o contexto em que surge determinado fator, algo apenas compreensível se acedermos à narrativa da trajetória idiossincrática de cada mulher. Efetivamente, a recuperação é encarada pelas ex-pacientes nos vários estudos mais como um processo do que como um estado final ${ }^{26}$. O estudo de fatores de recuperação de per si tem implicado nestes estudos o desmantelamento da recuperação, perdendo-se essa dimensão processual. Outra dificuldade de leitura desses fatores como promotores da mudança é, uma vez mais, o fato de essa busca ser desligada da compreensão da concepção que cada entrevistada tem do próprio problema/doença.

De fato, investigar sobre a recuperação é um tópico de alguma complexidade, por vários motivos. Primeiro porque a própria definição de recuperação tem sido de difícil consenso. Se alguns autores sugerem definições muito alargadas indo até o ponto de considerarem que a recuperação da anorexia nervosa implica a remissão de qualquer sintoma, abrangendo critérios mais globais de funcionamento, outros consideram que a definição de recuperação deve ser mais restrita e aplicar-se sempre que há remissão dos sintomas específicos da anorexia nervosa ${ }^{10}$. Essa dificuldade torna difícil a operacionalização de uma amostra, bem como o "retrato" de um fenômeno que pode variar consoante a definição que se emprega.

Em segundo lugar, tal como a trajetória da doença, a trajetória da recuperação é idiossincrática, o que faz com que, provavelmente, não haja uma única trajetória possível de recuperação. Poderão existir diferentes configurações de fatores, sendo claro que os mesmos fatores poderão ter sido úteis para umas e menos úteis para outras, dependendo da trajetória pessoal de cada uma.

Em terceiro lugar, a recuperação tem sido até agora estudada apenas a partir do ponto de vista da ex-paciente, apesar de, e da mesma forma que o fenômeno da doença, implicar possivelmente vários intervenientes (por exemplo, familiares, terapeutas, amigos). Estudos futuros poderiam lançar-se na compreensão do fenômeno da recuperação a partir não só do olhar da própria “ex-paciente", como também a partir de outros personagens que estiveram ativamente envolvidos no mesmo fenômeno.

Por último, parece haver em todos esses estudos o pressuposto de que a compreensão da recuperação se pode realizar independentemente do estudo da doença, como se a recuperação fosse a oposição clara à doença. Essa visão dualista poderá levar a uma definição de recuperação enquanto o regressar a um estado inicial existente antes do problema. Essa definição comporta a limitação de poder forçar uma correspondência entre o processo sintomático e o processo psicológico, pois se essa definição é verdadeira para a remissão de sintomas, já é perigosa do ponto de vista psicológico, em que um hipotético regresso ao estado inicial poderia corresponder a uma regressão. Em alternativa a essa visão dualista, seria interessante um olhar que contemplasse as continuidades e divergências como um processo que flui e evolui no eixo do tempo. Efetivamente, a polarização que se faz de doença e recuperação pode ser mesmo um obstáculo à mudança, já que inibe a negociação de significados da pessoa consigo própria e com as suas diferentes realidades. Se a recuperação for encarada como um aumento da liberdade da pessoa em relação a um problema que antes a asfixiava, é conveniente que na trajetória de mudança haja lugar para essa reautoria, que contempla flexibilidade e diversidade. Desse modo, o problema pode ser visto como uma potencialidade, e a recuperação, em vez de ser vista como cura ou resolução, poderá ser concebida como a "expansão dos processos de construção narrativa dos pacientes na direção de níveis mais sofisticados e complexos de expressão"30.

Em suma, apesar das limitações no nível da investigação das "diferenças que poderão fazer a diferença" na recuperação da anorexia nervosa, a tentativa de olharmos para a recuperação como um processo, de reautoria e negociação com o problema, no contexto das significações do próprio e dos que fazem parte dos seus nichos conversacionais mais significativos (pacientes, familiares, amigos, terapeutas), poderá constituir uma linha de investigação e, quem sabe, "uma diferença que possa fazer a diferença" na compreensão futura do processo de mudança.

Este trabalho faz parte de um projeto de investigação financiado pela Fundação para a Ciência e Tecnologia (Ref. Bolsa SFRH/ BD/27921/2006/J040276SBN7).

\section{Referências}

1. Kaplan AS. Psychological treatments for anorexia nervosa: a review of published studies and promising new directions. Can J Psychiatry. 2002;47(3):235-43.

2. Fairburn CG, Shaffran R, Cooper Z. A cognitive behavioural theory of anorexia nervosa. Behav Res Ther. 1999;37:1-13.

3. Eivors A, Button E, Warner S, Turner K. Understanding the experience of drop out from treatment of anorexia nervosa. Eur Eat Disord Rev. 2003;11:90-107.

4. Pike KM. Long-term course of anorexia nervosa: response, relapse, remission, and recovery. Clin Psychol Rev. 1998;19:773-88.

5. Roma Torres. Anorexia nervosa e perturbações afins do comportamento alimentar [dissertação não publicada]. Porto: Faculdade de Medicina da Universidade do Porto; 1986.

6. Anorexia Nervosa and Related Eating Disorders, Inc. [homepage]. Illinois: Treatment and recovery; 2005. Disponível em: <http://www.anred. com/tx.html>. Acesso em: 19 abr. 2008.

7. National Institute of Mental Health [homepage]. Eating disorders; 2005. Disponível em: <http://www.nimh.nih.gov>. Acesso em: 20 abr. 2008.

8. Herzog DB, Keller MB, Lavori PW. Outcome in anorexia nervosa and bulimia nervosa: a review of the literature. J Nerv Ment Dis. 1988;176:131-43.

9. Steinhausen HC. The outcome of anorexia nervosa in the 20th century. Am J Psychiatry. 2002;159(8):1284-93.

10. Couturier J, Lock J. What is recovery in adolescent anorexia nervosa? Int J Eat Disord. 2006;39(7):550-5.

11. Beresin EV, Gordon C, Herzog DB. The process of recovering from anorexia nervosa. J Am Acad Psychoanal. 1989;17:103-30.

12. Pinzon V, Nogueira FC. Epidemiologia, curso e evolução dos transtornos alimentares. Rev Psiq Clín. 2004;31(4):158-60. 
13. Fairburn CG, Harrison P. Eating disorders. Lancet. 2003;361:407-16.

14. Wilson GT, Vitousek KM, Loeb KL. Stepped care treatment for eating disorders. J Consult Clin Psychol. 2000;68:564-72.

15. Simmons D. Recovery from anorexia nervosa: a grounded theory study that looks at recovery from the perspective of the recovered anorexic [tese]. Capella University (UMI no 32066886); 2006.

16. Woods S. Untreated recovery from eating disorders. Adolescence. 2004;39(154):361-71

17. Garrett C. Recovery from anorexia nervosa: a sociological perspective. Int J Eat Disord. 1997;21:261-72.

18. Tozzi F, Sullivan P, Fear J, McKenzie J, Bulik C. Causes and recovery in anorexia nervosa: the patient's perspective. Int J Eat Disord. 2003;33:143-54.

19. Hsu LK, Crisp AH, Callender JS. Recovery in anorexia nervosa: the patient's perspective. Int J Eat Disord. 1992;11:341-50.

20. Maine M. An existential exploration of the forces contributing to, sustaining, and ameliorating anorexia nervosa: the recovered patient's view [tese]. Saybrook Institute; 1985.

21. Pettersen G, Rosenvinge JH. Improvement and recovery from eating disorders: a patient perspective. Eat Disord. 2002;10:61-71.

22. Latterza AR, Dunker KL, Scagliusi FB, Kemen E. Tratamento nutricional dos transtornos alimentares. Rev Psiq Clín. 2004;31(4):173-6.
23. Esherick M. How women recover from anorexia nervosa: an analysis of the recovery stories of 14 formerly anorexic women [tese]. Wright Institute Graduate Scholl of Psychology; 2003.

24. Federici A, Kaplan AS. The patient's account of relapse and recovery in anorexia nervosa: a qualitative study. Eur Eat Disord Rev. 2008;16:1-10.

25. Nillsson K, Hagglof B. Patient perspectives of recovery in adolescent onset anorexia nervosa. Eat Disord. 2006;14:305-11.

26. Matusek J. Overcoming an eating disorder: a narrative approach to long-term recovery [dissertação]. Ohio: Miami University; 2007.

27. Federici A. Relapse and recovery in anorexia nervosa: the patients' perspective. [dissertação]. Graduate Department of the Institute of Medical Sciences: University of Toronto; 2004.

28. Schoemaker $\mathrm{C}$. The principles of screening for eating disorders. In: Vandereycken W, Noordenbos G, editors. The prevention of eating disorders. New York: New York University Press; 1998, p. 187-213.

29. Liu A. Gaining: the truth about life after eating disorders. New York: Harper Collins; 2007.

30. Gonçalves O. Da psicopatologia como ficção à psicoterapia como criação: as más notícias. In: Gonçalves M, Gonçalves O, coordenadores. Psicoterapia, discurso e narrativa: a construção conversacional da mudança. Coimbra: Quarteto Editora; 2001, p. 65-90. 